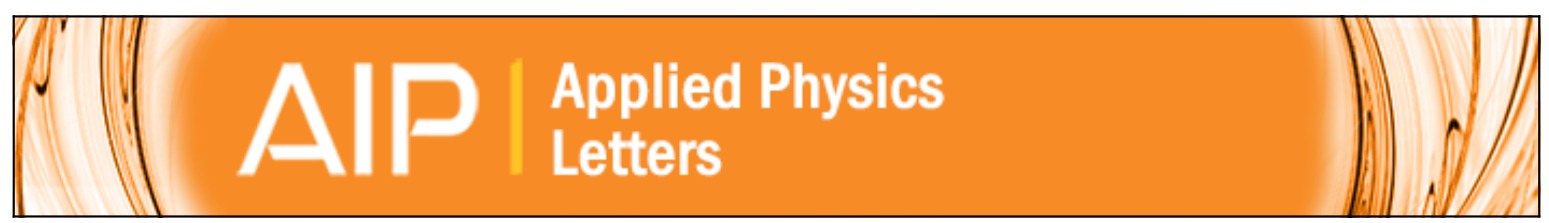

\title{
Ab initio study of noncovalent sidewall functionalization of carbon nanotubes
}

Seokho Lim and Noejung Park

Citation: Applied Physics Letters 95, 243110 (2009); doi: 10.1063/1.3274041

View online: http://dx.doi.org/10.1063/1.3274041

View Table of Contents: http://scitation.aip.org/content/aip/journal/apl/95/24?ver=pdfcov

Published by the AIP Publishing

\section{Articles you may be interested in}

Non-covalent functionalization of single wall carbon nanotubes and graphene by a conjugated polymer Appl. Phys. Lett. 105, 013103 (2014); 10.1063/1.4886968

Ab-initio calculations for a realistic sensor: A study of $\mathrm{CO}$ sensors based on nitrogen-rich carbon nanotubes AIP Advances 2, 032115 (2012); 10.1063/1.4739280

Ab initio study of semiconducting carbon nanotubes adsorbed on the Si(100) surface: Diameter- and registration-dependent atomic configurations and electronic properties

J. Appl. Phys. 100, 124304 (2006); 10.1063/1.2400404

$\mathrm{Ab}$ initio study of the effect of water adsorption on the carbon nanotube field-effect transistor Appl. Phys. Lett. 89, 243110 (2006); 10.1063/1.2397543

Soft-x-ray photoemission spectroscopy and ab initio studies on the adsorption of NO 2 molecules on defective multiwalled carbon nanotubes

J. Chem. Phys. 123, 034702 (2005); 10.1063/1.1947768

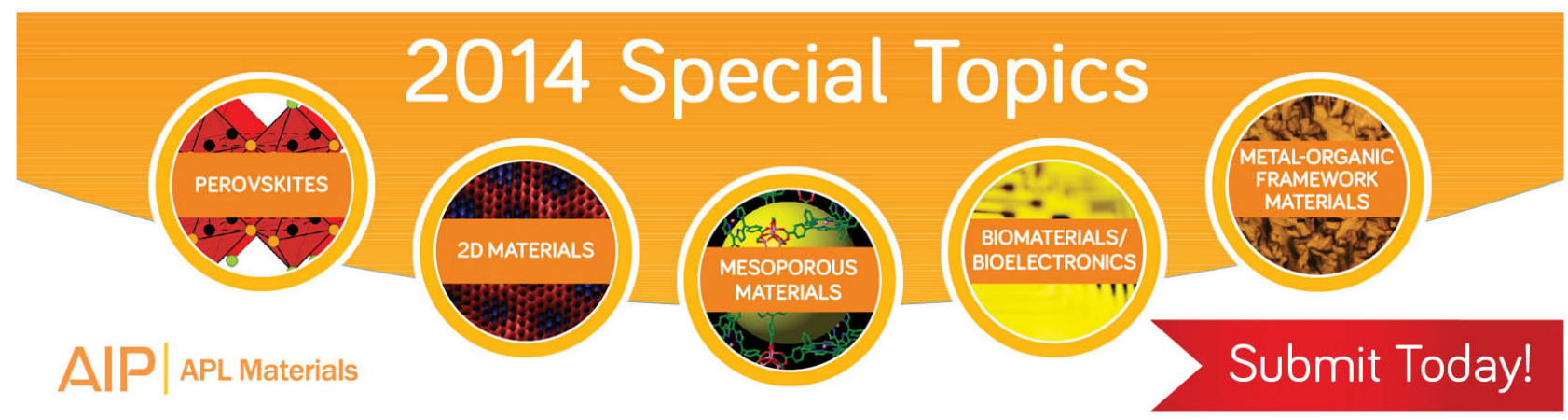




\title{
$A b$ initio study of noncovalent sidewall functionalization of carbon nanotubes
}

\author{
Seokho Lim and Noejung Park ${ }^{\mathrm{a})}$ \\ Department of Applied Physics, Dankook University, 44-1, Jukjeon-dong, Yongin-si, Gyeonggi-do 448-701, \\ Republic of Korea
}

(Received 11 August 2009; accepted 23 November 2009; published online 16 December 2009)

\begin{abstract}
We investigated noncovalent molecular adsorptions onto carbon nanotubes (CNTs), using density-functional theory methods including recently developed hybrid functionals. Planar aromatic molecules have greater binding strength than fully saturated nonaromatic molecules, and adsorption geometries are consistent with the features of intermolecular $\pi-\pi$ stacking. We show that modifications with alcohol or a thiol terminal group increase the adsorption strength consistently throughout the considered molecules. This result may be used in experiments involving noncovalent sidewall functionalizations of CNTs. (C) 2009 American Institute of Physics.

[doi:10.1063/1.3274041]
\end{abstract}

Methods for decorating carbon nanotubes (CNTs) with noncovalently adsorbed molecules, which is called noncovalent sidewall functionalization, have been desired for various purposes. Fabrication processes of high-strength fibers or other types of CNT composites are proceeded by the separation of as-grown aggregated CNTs. ${ }^{1-4}$ Aqueous solution of unharmed individual CNTs has been required for fundamental study of opto-electronic properties of the CNT. ${ }^{5,6}$ To attain these objectives, various methods of molecular adsorptions onto the outer surface of CNTs have been attempted. Among them, noncovalent methods are the most desirable because they are expected to preserve the mechanical and electronic advantages of CNTs. ${ }^{2,7}$ For example, researchers developing CNT-based biological sensors have sought moieties that have selective recognition capability for analytes and do not adversely affect the electronic structure of CNTs. ${ }^{8,9}$

In the present letter, we evaluate the underlying energetics of the noncovalent sidewall functionalization of CNTs. We performed density-functional theory (DFT) calculations to investigate the noncovalent binding energetics of molecules onto CNTs. ${ }^{10}$ We particularly focused on molecules used in experiments involving the noncovalent coating of CNTs. We show that the planar aromatic hydrocarbons have stronger binding affinity with the outerwall of CNTs than fully saturated nonaromatic hydrocarbons. The adsorption geometries are shown to be consistent with the known $\pi-\pi$ stacking interaction. ${ }^{11}$ In addition, we show that the introduction of terminal groups of alcohol or thiol moiety, as used in various experiments, increase the binding energetics of aromatic molecules onto CNTs. For computations, we used the Vienna $a b$ initio Simulation Package and the provided pseudoptentials. ${ }^{12,13}$ The local density approximation (LDA) and the generalized gradient approximation (GGA) were used for the exchange-correlation potential. ${ }^{14,15}$ The energy cut-off for the plane-wave basis was set to $400 \mathrm{eV}$. Geometry optimizations were carried out with residual forces of less than $0.01 \mathrm{eV} / \AA$. We particularly note that the DFT/ LDA can be the best choice for a practical evaluation of the

\footnotetext{
${ }^{\text {a) }}$ Author to whom correspondence should be addressed. Electronic mail: noejung@dku.edu. FAX: +82-31-8005-3208.
}

$\pi-\pi$ interaction (even better than the Moller-Plesset second order perturbation). ${ }^{16}$ Some of the M06 series of hybrid meta functions (M06-2X, M06-HF, and M06-L), which have been proven to have excellent capability for the $\pi-\pi$ stacking, were also employed for cross-checking. ${ }^{17}$ We used the NWChem package for actual calculations of the DFT hybrid functionals. ${ }^{18}$

Figure 1(a) shows the optimized geometry of the lowest energy structure of the pyrene-adsorbed $(10,0)$ CNT. A few metastable geometries were also found in our calculation with slightly higher energies. The stacking between the adsorbed pyrene and the CNT surface, as shown in Fig. 1(a), looks similar to the AB-stacked graphite. We note that this feature is consistent with the theory for the $\pi-\pi$ stacking, wherein the electrostatic attraction between $\sigma$ and $\pi$ electrons in the opposite molecules gives rise to a more or less strong intermolecular binding. ${ }^{11}$ The nearest $\mathrm{C}-\mathrm{C}$ distance, as denoted by the arrow in the inset of Fig. 1(a), was found to be $3.13 \AA$ in our DFT/LDA calculations. This clearly sug-

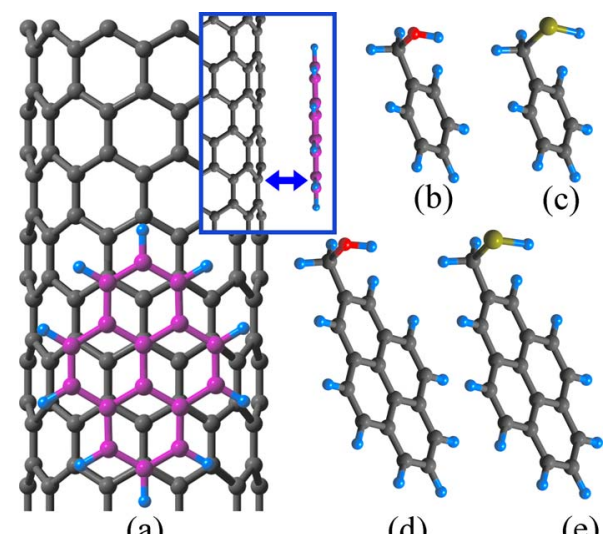

(a)

(d)

(e)

FIG. 1. (Color online) (a) Top view of optimized geometry of the lowest energy structure of the pyrene-adsorbed $(10,0)$ CNT. Inset shows the side view. Optimized geometries of (b) benzyl-alcohol, (c) benzyl mercaptan, (d) the thiol-terminated pyrene, and (e) the alcohol-terminated pyrene. In (a) to (e), the medium-sized balls and the smallest balls represent carbon and hydrogen atoms, respectively. The darker (red) medium-sized balls in (b) and (d) and the lighter (greenish yellow) larger balls in (c) and (e) denote oxygen and sulfur atoms, respectively. In the inset of (a), the nearest C-C distance between pyrene and the CNT is indicated with an arrow. 
TABLE I. Binding energy $\left[\mathrm{E}_{\mathrm{b}}(\mathrm{eV})\right]$ of aromatic molecules and diamondoids onto the surface of carbon nanotubes, calculated with the DFT with the LDA approximation.

\begin{tabular}{lcc}
\hline \hline & $(10,0) \mathrm{CNT}$ & $(5,5) \mathrm{CNT}$ \\
\hline Benzene $\left(\mathrm{C}_{6} \mathrm{H}_{6}\right)$ & 0.228 & 0.227 \\
Benzyl alcohol $\left(\mathrm{C}_{7} \mathrm{H}_{8} \mathrm{O}\right)$ & 0.270 & 0.273 \\
Benzyl mercaptan $\left(\mathrm{C}_{7} \mathrm{H}_{8} \mathrm{~S}\right)$ & 0.283 & 0.277 \\
Pyrene $\left(\mathrm{C}_{16} \mathrm{H}_{10}\right)$ & 0.455 & 0.429 \\
Pyrenyl alcohol $\left(\mathrm{C}_{17} \mathrm{H}_{12} \mathrm{O}\right)$ & 0.499 & 0.478 \\
Pyrenyl mercaptan $\left(\mathrm{C}_{17} \mathrm{H}_{12} \mathrm{~S}\right)$ & 0.490 & 0.476 \\
Adamantane $\left(\mathrm{C}_{10} \mathrm{H}_{16}\right)$ & 0.198 & 0.197 \\
Diamantane $\left(\mathrm{C}_{14} \mathrm{H}_{20}\right)$ & 0.204 & 0.229 \\
\hline \hline
\end{tabular}

gests that the adsorption of pyrene onto the CNT is a noncovalent interaction. Nevertheless, as will be shown in later paragraph, the binding strength for this type of $\pi-\pi$ interaction is noticeably stronger than the physisorption of nonaromatic molecules. ${ }^{2}$ To determine the size dependence of the binding mechanism and the effect of terminal group modifications, we performed similar calculations with benzene-derived and pyrene-derived molecules. Figures 1(b) and 1(c) show the optimized geometries of benzyl alcohol (BA) and benzyl mercaptan (BM), in which the alcohol and thiol moiety is decorated for a terminal group of benzene, respectively. The alcohol-terminated pyrene and thiolterminated pyrene, as shown in Figs. 1(d) and 1(e), respectively, were also considered. Hereafter, the alcoholterminated pyrene and the thiol-terminated pyrene are called pyrenyl alcohol (PA) and pyrenyl mercaptan (PM), respectively. In the present work, we are not concerned with whether or not PA and PM can be synthesized easily. Nonetheless, a comparison of them to BA and BM provides a good theoretical benchmark for the non-covalent functionalization of CNTs. It is noteworthy that the terminal groups of aromatic molecules have been manipulated in experiments involving CNT functionalizations. ${ }^{2,5,8}$

Our results for the binding energetics are compiled in Table I. We observe that the larger is the size of the adsorbed molecules, the stronger is the binding affinity with CNTs. This is consistent with common understanding of nonchemical intermolecular interaction. The binding energy naturally increases with the size of adsorbate molecules because the intermolecular physical interaction increases with the interfacial area between the two opposite molecules. However, such bindings are consistently stronger than physisorptions of nonaromatic molecules. For comparison, we calculated the binding energetics of the diamondoid molecules, in which all carbon atoms are interconnected through diamondlike tetrahedral bonds and surface carbon atoms are saturated with hydrogen atoms. ${ }^{19}$ Adamantane $\left(\mathrm{C}_{10} \mathrm{H}_{16}\right)$ and diamantane $\left(\mathrm{C}_{14} \mathrm{H}_{20}\right)$, as noted in Table I, are the two smallest diamondoids. The comparison between the binding energetics of aromatic molecules and diamondoids indicates that roughly $0.03-0.20 \mathrm{eV}$ could be attributed to the contribution from the $\pi-\pi$ stacking interaction.

The more noticeable feature is that the decoration of the terminal group with alcohol and thiol moiety increased the binding strength consistently throughout the considered molecules. The origin of the $\pi-\pi$ stacking interaction has been attributed to the electrostatic interaction between $\sigma$ electrons and $\pi$ electrons located in the opposite molecule,

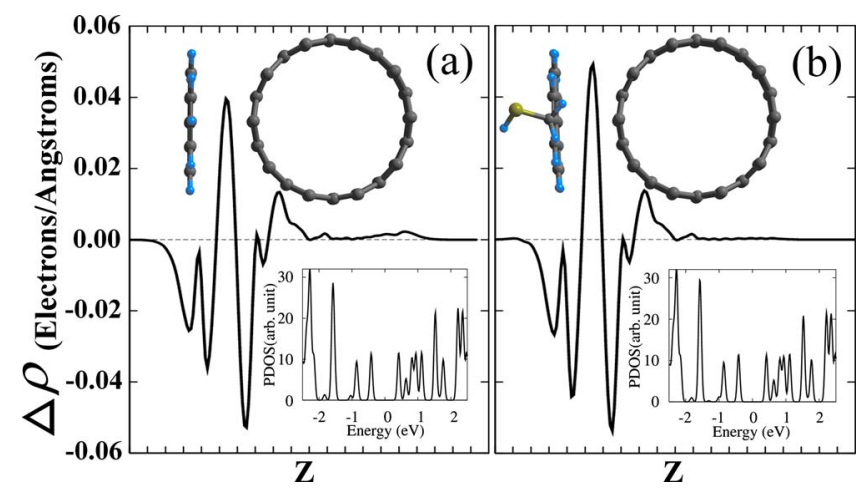

FIG. 2. (Color online) The change in electron density, $\rho(\mathrm{X} @ \mathrm{CNT})-\rho(\mathrm{X})-\rho(\mathrm{CNT})$, was averaged in the $x y$ plane and plotted along the $z$ axis, where (a) $\mathrm{X}$ is pyrene and (b) PM, respectively. Note that the aromatic molecule is parallel to the $x y$ plane. Insets show the partial density of states for the CNT in each case.

respectively. ${ }^{11}$ We conjecture that the alcohol or thiol terminal group may increase the polarity, thereby giving rise to an increment of the electrostatic interaction. In Fig. 2, we plotted the change in the total electron density along the perpendicular direction to the aromatic plane. We observe that the electron accumulation at the interface between PM and the CNT [Fig. 2(b)] is slightly larger than the electron accumulation between pyrene and the CNT [Fig. 2(a)]. However, as shown in the insets of Fig. 2, the electronic structures near the Fermi level are almost the same as that of the bare CNT. These features suggest that either pyrene or PM does not induce electron transfer, and the greater binding strength of $\mathrm{PM}$, in comparison with pyrene, can be attributed to the larger polarity incurred by electronegativity of PM.

It has been observed experimentally that $\pi-\pi$ stacking is so strong that the functionalized CNT compounds through the $\pi-\pi$ interaction can withstand heating up to a few hundred Celcius. $^{2}$ Of very significant interest is that the $\pi-\pi$ stacking does not destroy the electronic structure of the CNT despite its strong capability of functionalization. In the present work, we explicitly calculated the electronic structure of the CNTs with aromatic adsorbates to confirm the effect of $\pi-\pi$ stacking. The partial densities of electronic states of the $(10,0)$ CNT of the bare CNT geometry and the CNT with PM adsorbate are shown in Figs. 2(a) and 2(b), respectively. The two cases look almost identical, implying that the electronic structure of CNT remained intact over the adsorption of PM. We also investigated the other molecules, and produced almost the same results.

Because the nonexact nature of the exchange-correlation potential could be a source of error, results of DFT calculations should be interpreted with care. In previous paragraphs, we showed results of the DFT/LDA calculations and discussed that the $\pi-\pi$ interaction can be reinforced by a modification of terminal groups of the aromatic molecules. We particularly note that the LDA could be one of the best choice for the $\pi-\pi$ interactions, as demonstrated in the benchmark test by Zhao et al. ${ }^{16}$ In this paragraph, we show results of our calculations with the M06 series of hybrid meta functional to cross-check the LDA results. ${ }^{17}$ Through a series of case studies, the M06 suite of functionals were suggested to have decent accuracy for a diverse range of chemistry, particularly for the $\pi-\pi$ interaction. 


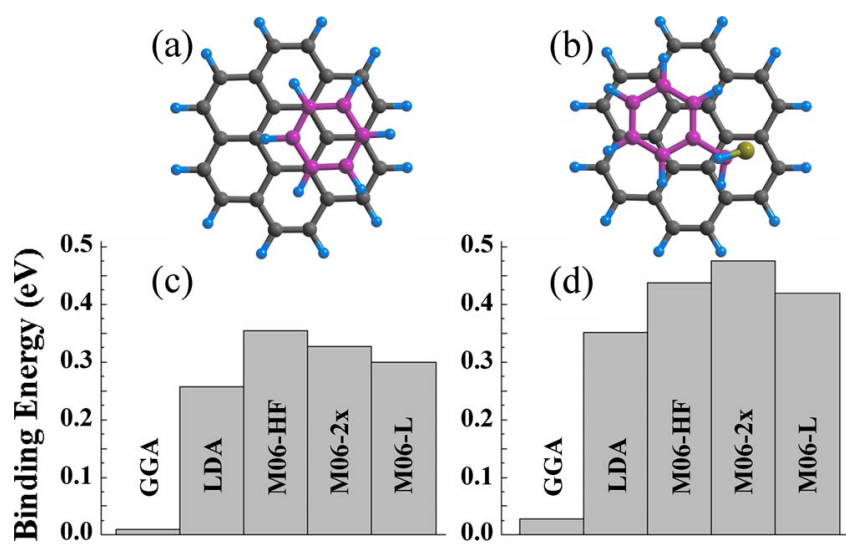

FIG. 3. (Color online) The equilibrium adsorption geometry of (a) benzene and (b) BM onto coronene. The colors of atomic symbols follows the same rule as in Fig. 1. The adsorption energy of (c) benzene and (d) BM onto coronene. For the computations with the M06 series we used the 6-311+ $+\mathrm{G}^{* *}$ basis set, and the basis set superposition errors were treated with the counterpoise correction.

Figures 3(a) and 3(b) show the lowest energy adsorption geometry of benzene and BM onto coronene. Figures 3(c) and 3(d) show the adsorption strength of benzene and BM onto coronene molecules, respectively. We observe that, irrespective of the computational method used, BM has greater binding strength than benzene. It has been discussed that practical implementations of DFTs cannot describe the $-1 / r^{6}$ behavior of long range interaction between neutral molecules ${ }^{20}$ whereas the M06 series of functionals could have some compensation for such a deficiency. However, the DFT/LDA and the M06 series are roughly consistent for equilibrium binding energetics of the $\pi-\pi$ interaction: BM has stronger binding affinity with coronene than benzene by about $0.11 \mathrm{eV}$, as presented in Fig. 3 .

In conclusion, using density-functional theory calculations, we investigated the binding energetics for noncovalent functionalizations of CNTs. Planar aromatic molecules have greater binding strength than fully saturated nonaromatic molecules. We showed that the modifications of aromatic molecules with alcohol or a thiol terminal group increased the $\pi-\pi$ stacking interaction with CNT surfaces. Using the LDA and some of recently developed hybrid functionals, we also showed that the modified terminal groups could enhance the $\pi-\pi$ attraction by about $0.11 \mathrm{eV}$.

The present research was conducted by the research fund of Dankook University in 2008.

${ }^{1}$ D.-Q. Yang, J.-F. Rochette, and E. Sacher, J. Phys. Chem. B 109, 4481 (2005).

${ }^{2}$ D.-Q. Yang, B. Hennequin, and E. Sacher, Chem. Mater. 18, 5033 (2006).

${ }^{3}$ P. Calvert, Nature (London) 399, 210 (1999).

${ }^{4}$ Y. Oh, D. Suh, Y. Kim, E. Lee, J. S. Mok, J. Choi, and S. Baik, Nanotechnology 19, 495602 (2008).

${ }^{5}$ N. Nakashima, Y. Tomonari, and H. Murakami, Chem. Lett. 31, 638 (2002).

${ }^{6}$ M. J. ÓConnell, S. M. Bachilo, C. B. Huffman, V. C. Moore, M. S. Strano, E. H. Haroz, K. L. Rialon, P. J. Boul, W. H. Noon, C. Kittrell, J. Ma, R. H Hauge, R. B. Weisman, R. E. Smalley, Science 297, 593 (2002).

${ }^{7}$ D. B. Farmer and R. G. Gordon, Nano Lett. 6, 699 (2006).

${ }^{8}$ R. J. Chen, Y. Zhang, D. Wang, and H. Dai, J. Am. Chem. Soc. 123, 3838 (2001).

${ }^{9}$ H. Dai, Acc. Chem. Res. 35, 1035 (2002).

${ }^{10}$ W. Kohn and L. J. Sham, Phys. Rev. 140, A1133 (1965).

${ }^{11}$ C. A. Hunter and J. K. M. Sander, J. Am. Chem. Soc. 112, 5525 (1990).

${ }^{12}$ G. Kresse and J. Furthmüller, Phys. Rev. B 54, 11169 (1996).

${ }^{13}$ G. Kresse and J. Furthmüller, Comput. Mater. Sci. 6, 15 (1996).

${ }^{14}$ J. P. Perdew, K. Burke, and M. Ernzerhof, Phys. Rev. Lett. 77, 3865 (1996).

${ }^{15}$ D. M. Ceperley and B. J. Alder, Phys. Rev. Lett. 45, 566 (1980).

${ }^{16}$ Y. Zhao and D. G. Truhlar, J. Phys. Chem. A 109, 5656 (2005).

${ }^{17}$ Y. Zhao and D. G. Truhlar, Acc. Chem. Res. 41, 157 (2008).

${ }^{18}$ E. J. Bylaska, W. A. de Jong, N. Govind, K. Kowalski, T. P. Straatsma, M. Valiev, D. Wang, E. Apra, T. L. Windus, J. Hammond, P. Nichols, S. Hirata, M. T. Hackler, Y. Zhao, P.-D. Fan, R. J. Harrison, M. Dupuis, D. M. A. Smith, J. Nieplocha, V. Tipparaju, M. Krishnan, Q. Wu, T. Van Voorhis, A. A. Auer, M. Nooijen, E. Brown, G. Cisneros, G. I. Fann, H. Fruchtl, J. Garza, K. Hirao, R. Kendall, J. A. Nichols, K. Tsemekhman, K. Wolinski, J. Anchell, D. Bernholdt, P. Borowski, T. Clark, D. Clerc, H. Dachsel, M. Deegan, K. Dyall, D. Elwood, E. Glendening, M. Gutowski, A. Hess, J. Jaffe, B. Johnson, J. Ju, R. Kobayashi, R. Kutteh, Z. Lin, R. Littlefield, X. Long, B. Meng, T. Nakajima, S. Niu, L. Pollack, M. Rosing, G. Sandrone, M. Stave, H. Taylor, G. Thomas, J. van Lenthe, A. Wong, and Z. Zhang, NWChem, A Computational Chemistry Package for Parallel Computers, Version 5.1 (Pacific Northwest National Laboratory, Richland, Washington, 2007), pp. 99352-0999; R. A. Kendall, E. Apra, D. E. Bernholdt, E. J. Bylaska, M. Dupuis, G. I. Fann, R. J. Harrison, J. Ju, J. A. Nichols, J. Nieplocha, T. P. Straatsma,T. L. Windus, and A. T.Wong, Comput. Phys. Commun. 128, 260 (2000).

${ }^{19}$ J. E. Dahl, S. G. Liu, and R. M. K. Carson, Science 299, 96 (2003).

${ }^{20}$ M. O. Sinnokrot and C. D. Sherrill, J. Phys. Chem. A 108, 10200 (2004). 